\title{
Laboratory measurements of geometrical effects in the $x$-ray emission of optically thick lines for ICF diagnostics
}

Cite as: Phys. Plasmas 26, 063302 (2019); https://doi.org/10.1063/1.5096972

Submitted: 21 March 2019. Accepted: 02 June 2019. Published Online: 18 June 2019

G. Pérez-Callejo (D, L. C. Jarrott (D, D. A. Liedahl, E. V. Marley, G. E. Kemp, R. F. Heeter (D) J. A. Emig, M. E. Foord, K. Widmann (D), J. Jaquez (D), H. Huang, S. J. Rose, J. S. Wark, and M. B. Schneider (D)

\section{ARTICLES YOU MAY BE INTERESTED IN}

Using L-shell x-ray spectra to determine conditions of non-local thermal dynamic equilibrium plasmas

Review of Scientific Instruments 89, $10 F 106$ (2018); https://doi.org/10.1063/1.5039357

The blind implosion-maker: Automated inertial confinement fusion experiment design Physics of Plasmas 26, 062706 (2019); https://doi.org/10.1063/1.5091985

Approaching a burning plasma on the NIF

Physics of Plasmas 26, 052704 (2019); https://doi.org/10.1063/1.5087256 


\title{
Laboratory measurements of geometrical effects in the X-ray emission of optically thick lines for ICF diagnostics
}

Cite as: Phys. Plasmas 26, 063302 (2019); doi: 10.1063/1.5096972

Submitted: 21 March 2019 - Accepted: 2 June 2019 •

Published Online: 18 June 2019

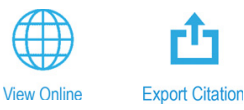

G. Pérez-Callejo, ${ }^{1, a)}$ (D L. C. Jarrott, ${ }^{2}$ (D) D. A. Liedahl, ${ }^{2}$ E. V. Marley, ${ }^{2}$ G. E. Kemp, ${ }^{2}$ R. F. Heeter, ${ }^{2}$ (DD J. A. Emig, ${ }^{2}$

M. E. Foord, ${ }^{2}$ K. Widmann, ${ }^{2}$ (iD J. Jaquez, ${ }^{3}$ (iD H. Huang, ${ }^{3}$ S. J. Rose, ${ }^{1,4}$ J. S. Wark, ${ }^{7}$ and M. B. Schneider ${ }^{2}$ (iD)

\author{
AFFILIATIONS \\ 'Department of Physics, Clarendon Laboratory, University of Oxford, Parks Road, Oxford OX1 3PU, United Kingdom \\ ${ }^{2}$ Lawrence Livermore National Laboratory, Livermore, California 94550, USA \\ ${ }^{3}$ Ceneral Atomics, San Diego, California 92121, USA \\ ${ }^{4}$ Plasma Physics Group, The Blackett Laboratory, Imperial College London, Prince Consort Road, London SW7 2AZ, United Kingdom \\ a) gabriel.perezcallejo@physics.ox.ac.uk
}

\begin{abstract}
Understanding the effects of radiative transfer in High Energy Density Physics experiments is critical for the characterization of the thermodynamic properties of highly ionized matter, in particular in Inertial Confinement Fusion (ICF). We report on non-Local Thermodynamic Equilibrium experiments on cylindrical targets carried out at the Omega Laser Facility at the Laboratory for Laser Energetics, Rochester NY, which aim to characterize these effects. In these experiments, a 50/50 mixture of iron and vanadium, with a thickness of $2000 \AA$ and a diameter of $250 \mu \mathrm{m}$, is contained within a beryllium tamper, with a thickness of $10 \mu \mathrm{m}$ and a diameter of $1000 \mu \mathrm{m}$. Each side of the beryllium tamper is then irradiated using 18 of the 60 Omega beams with an intensity of roughly $3 \times 10^{14} \mathrm{~W} \mathrm{~cm}{ }^{-2}$ per side, over a duration of $3 \mathrm{~ns}$. Spectroscopic measurements show that a plasma temperature on the order of $2 \mathrm{keV}$ was produced. Imaging data show that the plasma remains cylindrical, with geometrical aspect ratios (quotient between the height and the radius of the cylinder) from 0.4 to 2.0 . The temperatures in this experiment were kept sufficiently low $(\sim 1-2 \mathrm{keV})$ so that the optically thin Li-like satellite emission could be used for temperature diagnosis. This allowed for the characterization of optical-depth-dependent geometric effects in the vanadium line emission. Simulations present good agreement with the data, which allows this study to benchmark these effects in order to take them into account to deduce temperature and density in future ICF experiments, such as those performed at the National Ignition Facility.
\end{abstract}

Published under license by AIP Publishing. https://doi.org/10.1063/1.5096972

\section{INTRODUCTION}

The indirect drive approach to Inertial Confinement Fusion $(\mathrm{ICF})^{1-3}$ has experienced great progress over the past few years. ${ }^{4-6}$ To design more efficient experiments, a better characterization study of hohlraum plasmas in which the laser deposits its energy during the implosion is necessary. These plasmas are from the high- $\mathrm{Z}$ blow-off and gas-fill. However, obtaining accurate measurements of the plasma parameters inside the hohlraum in real time has proved to be a difficult challenge.

In particular, diagnosing the temperature is an important part of this study, as many of the relevant physical processes are temperature dependent. A more accurate measurement of the temperature leads to better measurements of indirect properties and to a better understanding of the overall plasma conditions. A widely used method for temperature diagnostics in Plasma Physics is the analysis of the ratio of intensities between different spectral lines. ${ }^{9-}$

The He- $\alpha$ emission complex $\left(1 \mathrm{~s} 2 \mathrm{l} \rightarrow 1 \mathrm{~s}^{2}\right)$ presents several lines with similar photon energies but different line strengths, which make it highly interesting for characterizing the plasma conditions. ${ }^{14}$ It is made up of four lines, namely, the resonance or $w$ line $\left(1 \mathrm{~s} 2 \mathrm{p}{ }^{1} \mathrm{P}_{1}\right.$ $\left.\rightarrow 1 \mathrm{~s}^{2}{ }^{1} \mathrm{~S}_{0}\right)$, two intercombination lines, the $x$ line $\left(1 \mathrm{~s} 2 \mathrm{p}^{3} \mathrm{P}_{2} \rightarrow 1 \mathrm{~s}^{2} \mathrm{~S}_{0}\right)$ and the $y$-line $\left(1 \mathrm{~s} 2 \mathrm{p}^{3} \mathrm{P}_{1} \rightarrow 1 \mathrm{~s}^{2}{ }^{1} \mathrm{~S}_{0}\right)$, and the forbidden or $z$ line (1s $2 \mathrm{~s}$ ${ }^{3} \mathrm{~S}_{1} \rightarrow 1 \mathrm{~s}^{2}{ }^{1} \mathrm{~S}_{0}$ ). We are using here the notation given by Gabriel. ${ }^{15}$ Additionally, emission from Li-like satellite levels ( $\left.1 \mathrm{~s} 2 \mathrm{l} n \mathrm{l}^{\prime} \rightarrow 1 \mathrm{~s}^{2} n \mathrm{l}^{\prime}\right)$ is usually blended with the $\mathrm{He}-\alpha$ complex. 
In 1969, Gabriel and Jordan ${ }^{16}$ developed a method for diagnosing the temperature of coronal plasmas using the ratio $(x+y+z) / w$. This method has been widely used in astrophysical ${ }^{17-23}$ and laboratory plasmas $^{24}$ over the past few decades. However, this method cannot be applied at the high densities found in High Energy Density (HED) plasmas since the $x$ and $z$ lines are not present owing to the high collisionality of the system. This is shown in Fig. 1, where we compare simulated spectra of He-like vanadium for two different ion densities (the temperature in both cases is $600 \mathrm{eV}$ ). One can see that the contribution from the $x$ and $z$ lines vanishes at HED conditions, with the $y$ and $w$ lines being the main components. In these conditions, comparison between the same complex for different elements (isoelectronic lines) $)^{25-27}$ or between $\mathrm{He}$ and $\mathrm{H}$-like lines ${ }^{27-30}$ is often used instead. When comparing the emission from $\mathrm{He}$ and $\mathrm{H}$-like ions, higher series lines (such as $\beta$ and $\gamma$.) are commonly preferred, given that most experimental designs render them optically thin.

One way to use this method in HED experiments is the so-called Dot Spectroscopy approach, developed by Apruzese. ${ }^{29,30}$ It consists of placing a microdot of a mid- $Z$ element $(Z \sim 20-30)$ in the HED plasma so that it thermalizes with it and presents K-shell emission. This approach was used at NOVA ${ }^{31}$ in its early stages of development ${ }^{26,32}$ and has been used more recently at the National Ignition Facility (NIF) ${ }^{33}$ to obtain the time evolution of the temperature of the hohlraum plasma during an ICF experiment. ${ }^{27,34}$

Most of the experiments using this approach ${ }^{27,32,34}$ at the National Ignition Facility (NIF) focus on the He- $\alpha$ emission, as it is one of the most intense components of the spectrum. One problem when using the He- $\alpha$ complex is that the cylindrical geometry of the dots' plasma affects the angular distribution of optically thick lines

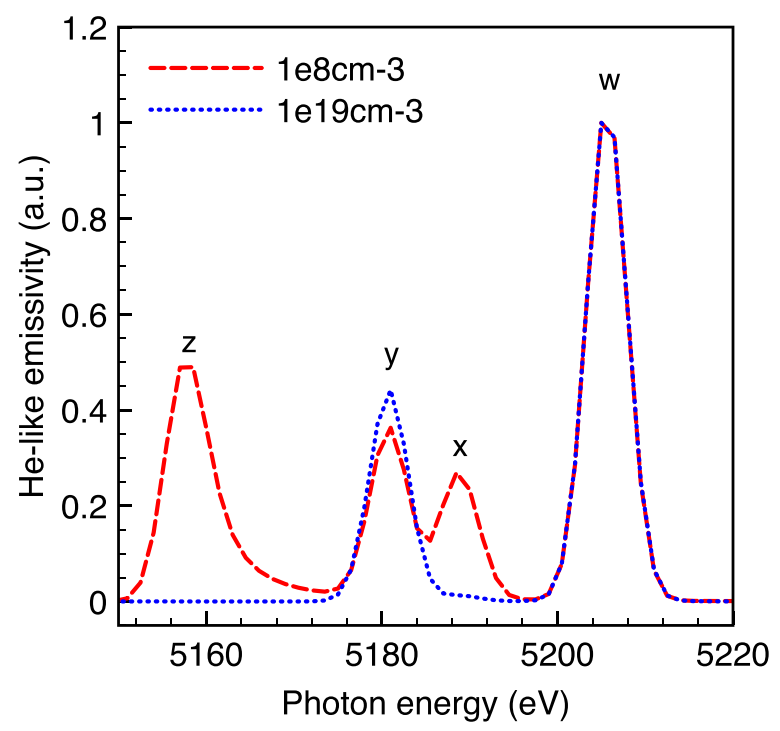

FIG. 1. Comparison between the emission from He-like $V(Z=23)$ for different conditions. The dashed red line corresponds to the conditions studied by Gabriel and Jordan $^{16}\left(n_{i}=10^{8} \mathrm{~cm}^{-3}\right)$, whereas the blue dotted line corresponds to $n_{i}$ $=10^{19} \mathrm{~cm}^{-3}$. Both simulations correspond to an electron temperature $T=600 \mathrm{eV}$. Note how the $x$ and $z$ lines are present in coronal conditions, but they disappear for higher ion densities. The Be-like and Li-like contributions have been removed for clarity. through the optical depth (Back, in $1996,{ }^{26}$ used the He- $\beta$ emission instead, which is not affected by the geometry, but is less intense).

The effects of the plasma geometry on the spectrum of optically thick lines are a widely known effect in astrophysics. Bhatia and coworkers $^{35-37}$ showed that under some circumstances, the emission of an optically thick line can be enhanced with respect to the optically thin limit. Later, Kerr et al. ${ }^{38-40}$ proved that this effect was related to the ratio between the Line Of Sight (LOS) through the target in the direction of the detector and the mean chord of the plasma. This work considered the ratio of two distinct spectral lines in plasmas, uniform in density and temperature but of differing geometries, which are close to coronal (or at least semicoronal) conditions. The opacity effect exhibits itself in the observed ratio of these two spectral lines that traverse the same physical path length (the LOS). Along this length one of the lines is thought to be optically thick, and the other optically thin. Kerr et al. studied the effect of opacity by calculating an intensity ratio for the optically thick line, $f=I_{\text {thick }} / I_{\text {thin }}$, where $I_{\text {thick }}$ is the intensity of this line when calculated including opacity effects, and $I_{\text {thin }}$ is the intensity of the "very same line," calculated neglecting such opacity effects both on the populations and in line transfer. The ratio $f$ is not of itself a directly experimentally observable for a given line, as a particular line is either thick or thin. However, whatever the particular species present within a plasma, the ratio $f$ can always be directly related to an experimentally observable ratio between "different" spectral lines, where one of them is optically thin, simply by multiplying with a known constant-and this is the role played by the second (optically thin) line, referred to above. Interestingly, depending upon the ratio of the mean chord to the LOS, $f$ can be greater or less than unity-that is to say that the intensity of an optically thick line can be larger than what one would expect had opacity effects been neglected in the calculation. This will occur with mean chords larger than the LOS, such that the effect of opacity is to increase the population of the upper level of the state associated with the emitted line radiation. Conversely, if the LOS is much greater than the mean chord, $f$ can be less than unity, and effective suppression of the optically thick line occurs. Given that the above effect depends on the mean chord (which can be obtained by means of the Dirac-Fuchs theorem ${ }^{41,42}$ ) and LOS lengths, this effect is what we call geometric anisotropy and has been previously used to characterize the geometry of astrophysical bodies. ${ }^{40,43}$ It has also been observed in HED experiments, ${ }^{44}$ and further experiments to explore it have been proposed. ${ }^{40}$ In the experiments described here the optically thick line, for which we seek to observe these opacity effects, is the resonance $(w)$ line of the $\mathrm{He}-\alpha$ complex of $\mathrm{V}$, and the optically thin line to which we reference its intensity is the associated intercombination $(y)$ line.

In the case of dot spectroscopy, the dot's plasma has a cylindrical shape. For this reason, depending on its aspect ratio, $H / R$, optically thick lines will be enhanced or suppressed depending on the LOS of the detector. An example of the angular distribution of radiation from an optically thick line is shown in Fig. 2. This case corresponds to a cylinder with a $H / R$ ratio of $2 / 3$. The optical depth of the optically thick line in the radial direction is 10 . In this example, the axial emission (small angles) corresponds to the short dimension, whereas radial emission (high angles) corresponds to the long dimension of the cylinder. Accounting for these effects on the line ratios is essential to obtain accurate measurements of the plasma temperature and density in nonspherical plasmas.

The optical depth of a line transition with frequency $\nu$ in a plasma of characteristic length $L$ is 


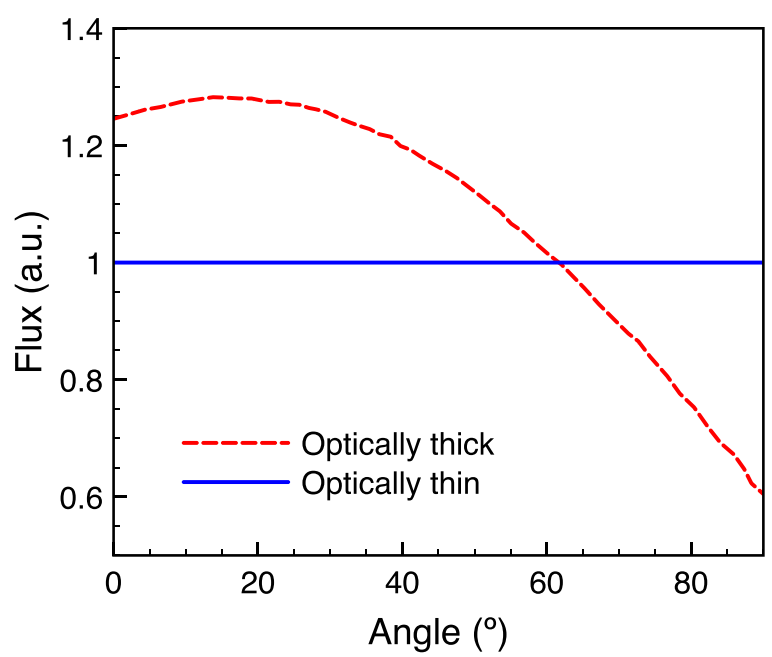

FIG. 2. Distribution of the spectral flux in a plasma cylinder for an optically thick and an optically thin line as a function of the angle with respect to the cylinder axis (calculations done by the authors). The aspect ratio, the ratio of the cylinder's height to radius, $H / R$ in this case is $2 / 3$. For this particular example, the optical depth in the radial direction is 10 . The distribution for the optically thin line is independent of the angle and corresponds to a spherically symmetric plasma, whereas the emission from optically thick lines depends on the angle of observation.

$$
\tau_{\nu}=n_{a} L \sigma(\nu),
$$

where $n_{a}$ is the density of absorbers (that is the density of ions in the ground state of the transition) and $\sigma$ is the resonant line photoabsorption cross section, on which the effect of the geometric anisotropy depends. The cross section is given by

$$
\sigma(\nu)=\frac{\pi e^{2}}{m_{e} c} f \phi(\nu),
$$

where $f$ is the oscillator strength of the transition and $\phi(\nu)$ is the line absorption profile. The oscillator strength of the $y$-line is $\sim 10-20$ times lower than that of the $w$-line for mid-Z elements. For this reason, while the $y$-line is optically thin, and therefore is not affected by the plasma geometry and can act as our reference as described above, the $w$-line is optically thick, and the geometric anisotropy effect must be taken into account to analyze its angular radiation pattern.

We present an experimental platform to characterize the effects of the geometry on the radiation pattern of optically thick lines in uniform cylindrical HED plasmas. We have characterized the temperature and density of the plasma from its spectrum and pinhole imaging. The $w / y$ ratios were obtained from the spectra as a function of the aspect ratio of the cylinder (height/radius). We have directly measured $\mathrm{X}$-ray line intensity enhancements and suppressions as a function of the plasma geometry. Full radiative transfer simulations agree with the experimental data. This work helps us to better understand HED plasmas and to obtain more information from their spectra.

\section{EXPERIMENTAL SETUP}

The experiments were performed at the OMEGA laser facility $^{45}$ at the Laboratory for Laser Energetics (LLE). The targets were tamped disk-shaped foils consisting of an inner foil made of an iron and vanadium mixture, volumetrically equal, surrounded by a beryllium tamper. The inner sample material was $0.2 \mu \mathrm{m}$ thick and $250 \mu \mathrm{m}$ in diameter. The outer tamper was $10 \mu \mathrm{m}$ thick ( $5 \mu \mathrm{m}$ on either side of the sample), with an outer diameter of $1000 \mu \mathrm{m}$. Having a larger tamper radius allows for a greater amount of $\mathrm{Be}$ outside the sample radius to provide radial confinement via thermal pressure. ${ }^{46}$ The surface of the targets was smoothed to obtain a more uniform ablation surface, which means that the conduction layer will reach the sample material evenly and keep the sample temperature as uniform as possible, preventing hydrodynamic instabilities. 18 of the 60 OMEGA laser beams at $3 \omega$ were irradiated on each surface, with an intensity of $\sim 3 \times 10^{14} \mathrm{~W} \mathrm{~cm}^{-2}$ per side, or $\sim 6 \times 10^{14} \mathrm{~W} \mathrm{~cm}^{-2}$ when the laser burns through, with a total laser energy of $\sim 10 \mathrm{~kJ}$. A schematic diagram of the experimental setup is shown in Fig. 3.

During the design of the experimental platform, twodimensional HYDRA ${ }^{47}$ simulations were run to estimate the degree to which the Fe:V plasma would remain uniform. It was found that $\sim 2$ ns into the pulse, the Fe:V plasma becomes underdense and the laser heats the entire plasma volume. At this point, the Fe: $\mathrm{V}$ plasma becomes roughly isothermal, although with density gradients mostly in the radial direction, causing differences in density up to a factor of four. $^{48}$

Time-resolved K-shell spectral emission from the Fe:V plasma was obtained using two Multipurpose Spectrometers (MSPECs), each looking at the emission from either Face-on or Side-on view (axial and radial emission, respectively). These instruments obtained spectra in four time windows, each one integrated over 200 ps and with a separation of $400 \mathrm{ps}$. The time-resolved axial and radial expansion was measured using two X-Ray Pinhole Cameras (XRPINH), one for each view. The expansion was obtained in sixteen 50 ps time windows.

The OMEGA chamber has a truncated icosahedron geometry (soccer-ball). Ports for diagnostics are at the center of the flat hexagons
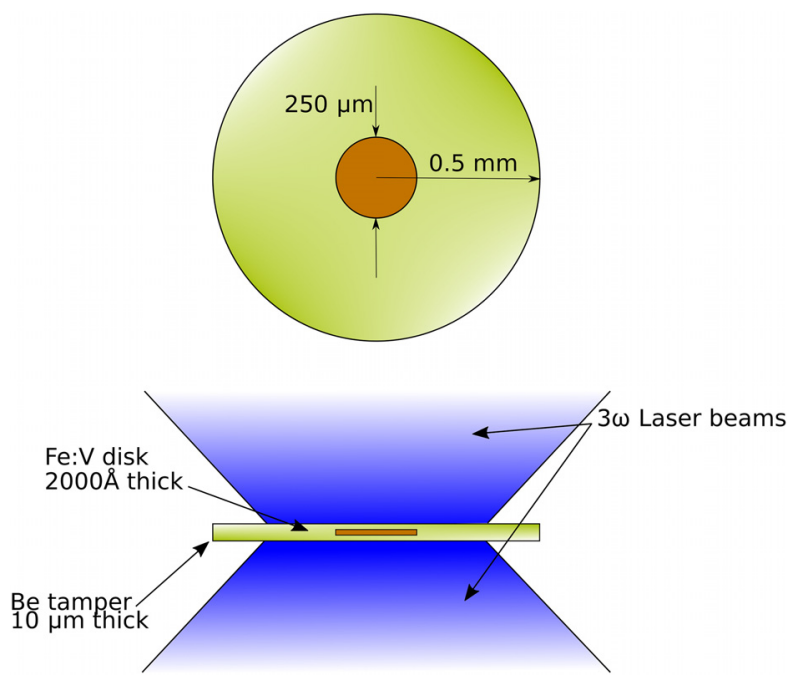

FIG. 3. Front (top) and side (bottom) views of the targets. The $250 \mu \mathrm{m}$ diameter disk is a mixture of $\mathrm{Fe}$ and $\mathrm{V}(1000 \AA \mathrm{A}: 1000 \AA)$ tamped in a $10 \mu \mathrm{m}$ thick $1 \mathrm{~mm}$ diameter Be disk. 36 laser beams impinge upon the target, 18 on each side, heating them up to $\sim 2 \mathrm{keV}$ in $3.0 \mathrm{~ns}$. The thickness of the buried disk has been exaggerated for clarity. 
or pentagons. There are six Ten-Inch Manipulator (TIM) ports, in which various cameras and snouts can be loaded, moved into the target chamber at desired distance from the center, and pointed. Of the six TIM ports, four are in two pairs, such that the ports in each pair are opposing each other (TIM 2 and 3, and TIM 4 and 6). Each pair housed one framing camera with a MSPEC snout and one framing camera with a XRPINH snout, ${ }^{49}$ so both diagnostics had the same view.

To minimize measurement uncertainties due to differences in individual diagnostic sensitivities, two target orientations (TOs) were used on alternating shots. This allowed each crystal spectrometer and pinhole camera to have both Face-on and Side-on views of the target, eliminating systematic errors due to crystal imperfections or framing camera sensitivities. At the same time, this configuration minimizes the timing uncertainty, as the jitter for each MSPEC and XRPINH with respect to the start of the laser pulse is consistent, whereas it changes between diagnostics. The TOs were calculated such that in one case (TO1) the target normal was perpendicular to TIMs 2 and 3, whereas in the other (TO2) it was perpendicular to TIMs 4 and 6. In both cases, the TO was such that the Face-on view was as close to normal as possible $\left(10.8^{\circ}\right)$. The Target Normals (TNs) were calculated for each TO as

$$
\begin{aligned}
& \mathrm{TN}_{1}[\theta, \phi]=\left(\mathrm{T}_{2} \times \mathrm{T}_{4}\right) \times \mathrm{T}_{2}=\left[52.64^{\circ}, 341.98^{\circ}\right], \\
& \mathrm{TN}_{2}[\theta, \phi]=\left(\mathrm{T}_{4} \times \mathrm{T}_{2}\right) \times \mathrm{T}_{4}=\left[26.57^{\circ}, 161.97^{\circ}\right],
\end{aligned}
$$

where $\theta$ and $\phi$ are the polar and azimuthal angles of the OMEGA target chamber and $\mathrm{T} 2$ and $\mathrm{T} 4$ are the diagnostic port orientations of TIM2 and TIM4, respectively (where the MSPECs were mounted). Data in both TOs from the same MSPEC were compared, which minimizes the systematic error in the line ratios from the instrument response. This is valid because the TOs were equivalent and the OMEGA Laser is highly reproducible (as verified in the experiment). Figure 4 shows the view of each instrument for both TOs.

Thirty-six of the $3 \omega$ laser beams were used, with 18 of them impinging upon each face of the targets. The laser profile was designed so that $90 \%$ of the laser intensity was deposited on a circle with twice the diameter of the Fe: $\mathrm{V}$ disk, thus minimizing edge effects in the heating process of the Fe: $\mathrm{V}$ plasma. Also, heating the outer tamper implies that it will create a radial pressure to the buried sample, which improves the radial confinement. In order to maintain nominally similar on-target drive, beams were selected for each TO to match the overall intensity and laser footprint, while also meeting facility beam safety requirements. To match the intensity and overall footprint, the average incident beam angle with respect to the target normal, as well as the variance in the incident beam angle for each TO, was matched. Calculations prior to the experiment predicted an agreement between the nominal drive for both TOs within $0.16 \%$. The laser configuration for each TO, as well as the residual between them, is shown in Fig. 5. In this figure, the inner solid red circle represents the Fe: $\mathrm{V}$ disk, whereas the dashed line corresponds to twice its diameter. It can be seen how there are no significant intensity differences in the sample.

\section{A. Validation of target orientation}

The consistency between the two target orientations was checked experimentally using two diagnostics. A p510 optical streak camera measured the total power profile and indicated that it was similar for

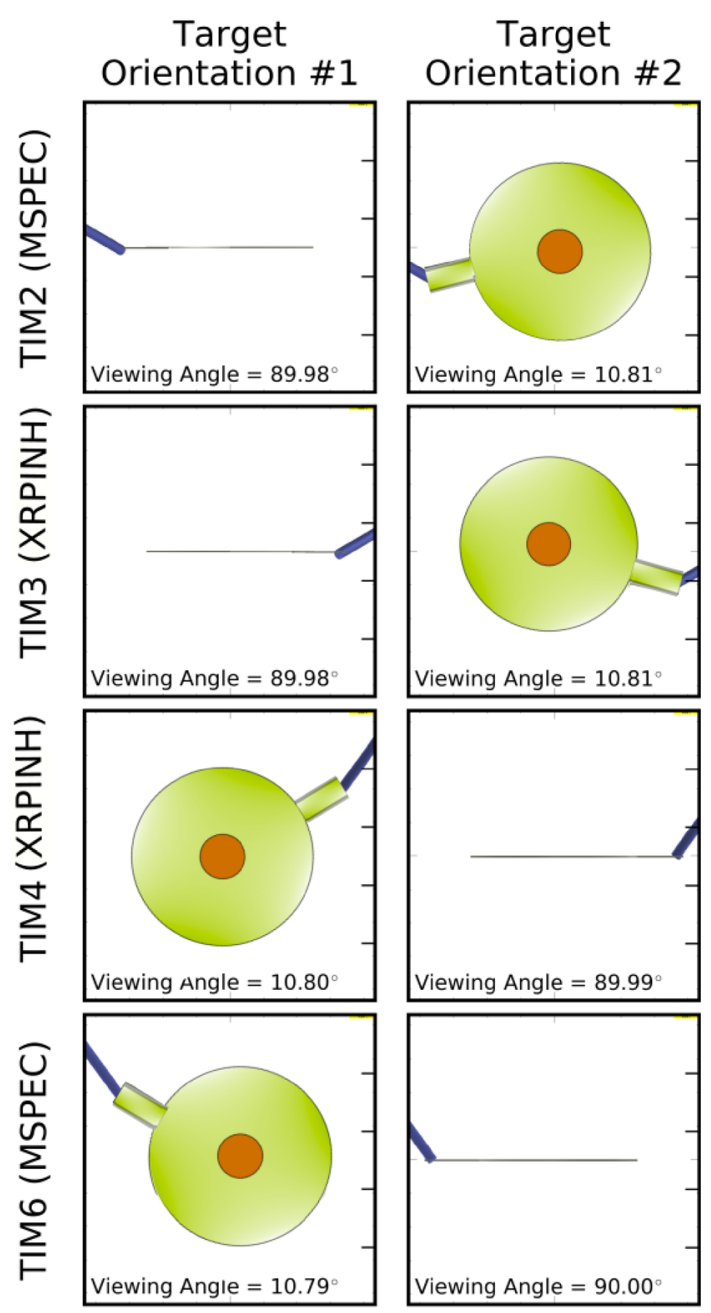

FIG. 4. Different views for all crystal spectrometers (MSPEC) and pinhole imagers (XRPINH) for the different TOs. Each column corresponds to one of the TOs, and each row to diagnostics. The indicated viewing angle is measured with respect to the target normal. Using this configuration it is possible to eliminate instrumental effects in the Face-on and Side-on data.

both TOs, given the shot to shot variation, with peak differences of less than $\pm 20 \%$.

A second consistency check was done using the diagnostic DANTE. ${ }^{50-52}$ This instrument is an absolutely calibrated diode-array spectrometer. It has 18 channels obtaining the time-resolved absolute flux emitted by the target, each channel observing a different X-ray energy range. In this experiment, there were 14 active channels, covering photon energies from 50 to $20000 \mathrm{eV}$. For both TOs, the angle of the diagnostics with respect to the target normal was $29^{\circ}$. The absolute fluxes emitted for both TOs show good agreement (within 10\%) at all times during the emission, thus showing that both laser configurations drive the targets in a similar way. Figure 6 shows an example of this comparison, corresponding to a signal measured by channel 9 of DANTE $(h \nu=1.3-1.5 \mathrm{keV})$, which measures the L-shell emission of the plasma. 

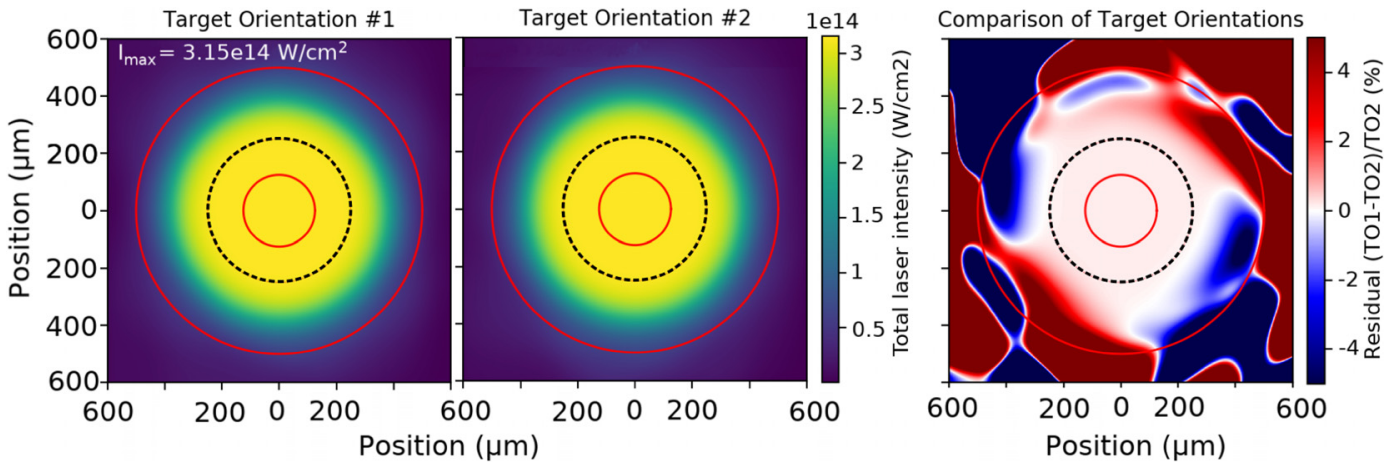

FIG. 5. Simulations of the laser drive on target for both TOs. The outer solid circle corresponds to the diameter of the Be tamper, whereas the inner solid circle corresponds to the diameter of the Fe:V disk. The laser profile was designed so that $90 \%$ of the laser intensity was deposited in a circle of twice the diameter of the buried disk (dashed black line) to minimize edge effects. The plot on the right shots the percentual residual between the drives for each configuration.

\section{RESULTS}

High quality spectra were obtained from these experiments. In Fig. 7, we have plotted the MSPEC and XRPINH data from two different shots representing an experimental "pair" of shots which had identical drive parameters with the exception of target orientations. The left two columns are Side-on data from Target Orientation 1 (TO1) while the right two columns are Face-on data from Target Orientation 2 (TO2). The MSPEC data shown in columns 1 and 3 in Fig. 7 are consequently taken from the same diagnostics, meaning that the diagnostic timing with respect to the start of the driver beams is nominally identical. Additionally, since the same MSPEC is shown in both columns, the crystal reflectivity and the overall diagnostic response are identical making direct comparisons between them straightforward. Likewise, the XRPINH data shown in columns 2 and 4 are also from the same

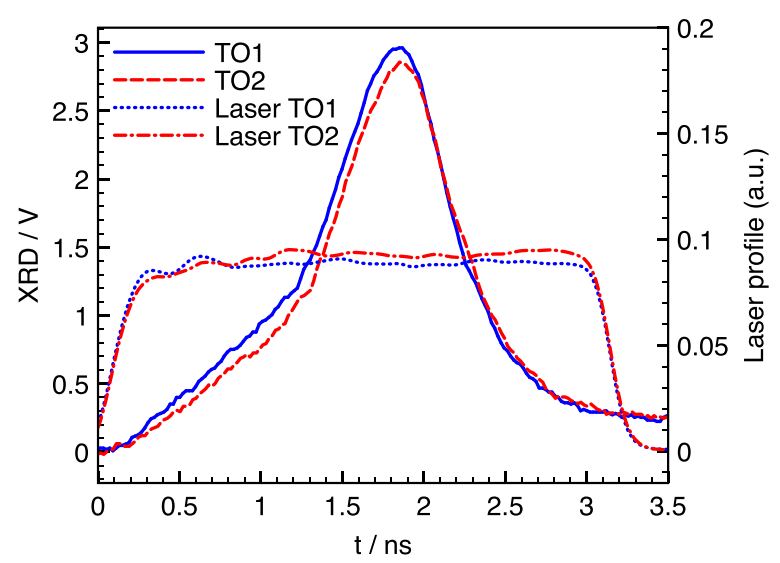

FIG. 6. Comparison between the total power (in units of voltage measured by DANTE) emitted by the plasma in the 1.3-1.5 keV photon energy range for both TOs. It can be seen that in both TOs, the emitted power is very similar, which is indicative of the fact that the shots are equivalent and therefore, they can be compared. The $x$-axis corresponds to the time with respect to the start of the laser pulse. The $y$-axis shows the signal measured by the X-ray Diode (XRD) in Volts. The angle of view for both TOs is $29^{\circ}$. The laser profile for both TOs is also shown in arbitrary units for comparison. diagnostics, and therefore, the target geometry from Face-on and Side-on views is shown at identical times with respect to the start of the laser drive for each respective shot. The spectra are normalized to the peak of the $y$-line, as the detector is not calibrated.

The rows in Fig. 7 correspond to different frames from all four diagnostics, with time going down. The first row was taken at $1.9 \mathrm{~ns}$ after the start of the driver beams, the second row at $2.3 \mathrm{~ns}$, the third at $2.7 \mathrm{~ns}$, and the fourth at $3.1 \mathrm{~ns}$, just after the laser is turned off (at $3.0 \mathrm{~ns}$ ). In the XRPINH data, there is a density gradient in the radial direction, which is most intense between the first and the second rows. This behavior is in agreement with the predictions from the hydrodynamic simulations.

We will not account here for the iron emission, as there was a significant amount of iron in the Be tamper, which blends with the emission from the disk. The spectral data in columns 1 and 3 show only the Vanadium He- $\alpha$ complex. Since the $y$-line $(\sim 5185 \mathrm{eV})$ is optically thin, whereas the $w$-line $(\sim 5210 \mathrm{eV})$ is not, it is possible to quantify the anisotropy effect due to target geometry by comparing the $w / y$ ratio at each time. The spectra have been normalized to the peak of the $y$-line for ease.

It can be seen that the spectra from Face-on and Side-on view differ in the relative intensity of the $w$ and $y$ lines, whereas the relative intensity of the $y$-line and the Li-like satellite peak $(\sim 5165 \mathrm{eV})$ is independent of the view. Figure 8 shows a particular example of these data. The spectra correspond to $t=2.3 \mathrm{~ns}$ after the start of the pulse, and the aspect ratio $H / R$ (defined as the ratio of the height along the axial direction to the radius of the cylinder) at that time is 0.82 . Here, the $w$ line is significantly more intense than the $y$-line in the Face-on view (column 3 in Fig. 7), whereas in the Side-on view (column 1), both lines are comparable. This is indicative of the fact that a significant amount of resonant line photons leave the plasma along the shortest escape path (Face-on). As the aspect ratio increases (third and fourth rows in Fig. 7), this effect becomes less important and the differences between both views are reduced.

\section{A. Target expansion}

The size of the plasma was obtained from the XRPINH images (second and fourth columns in Fig. 7). Lineouts through the centroid of each image were fitted to supergaussians. A combined analysis of 
Side-on
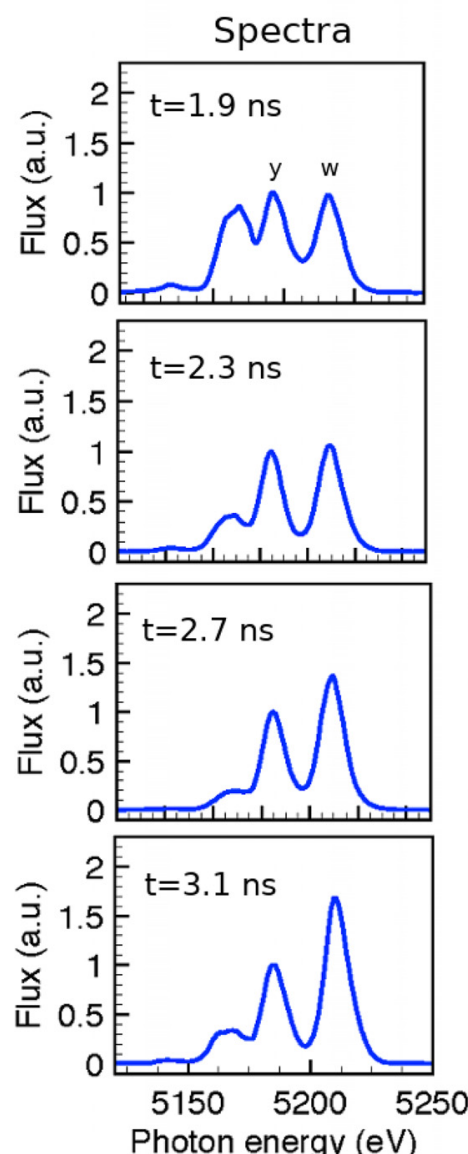

Face-on Imaging
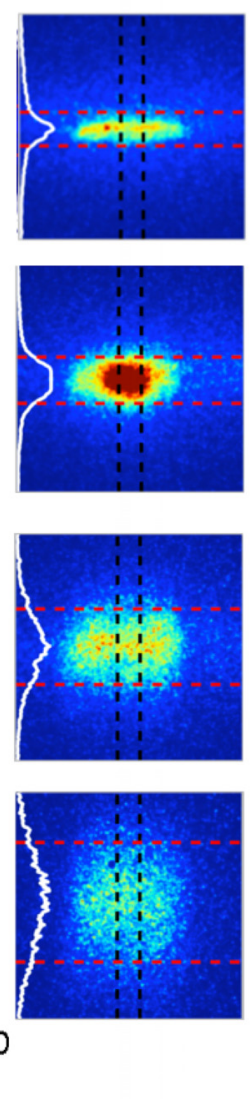

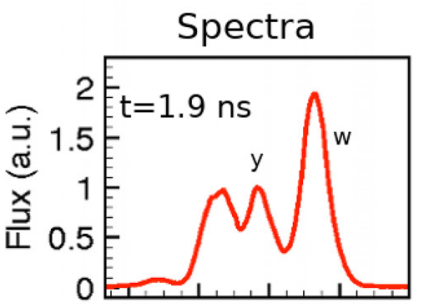

Imaging
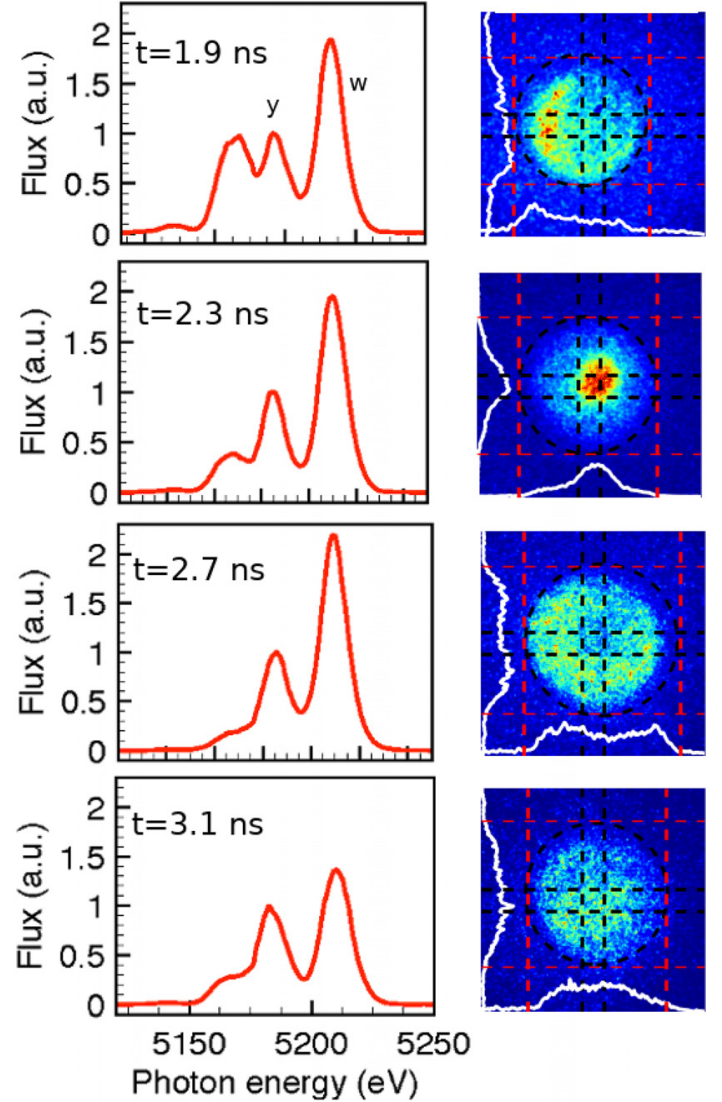

FIG. 7. Spectral data and imaging for Side-on and Face-on views. Each row represents a time step in the plasma expansion, and time goes from top to bottom. The spectra are normalized to the peak of the $y$-line. Note how the w/y ratios are different for each view at each time step. The bumps on the low-energy wing of the $y$-line correspond to the Li-like satellite emission. For a given view, as the plasma expands the ratios change as a function of time. Note that the expansion occurs mostly in the axial direction (side-on imaging) whereas the radius stays relatively constant for every time step. The imaging data are normalized to their peak at every time step. The red dashed lines represent the measured limits of the plasma in both dimensions. The white lines show the lineouts through the center of the images, whereas the dashed black lines represent the regions used to integrate those lineouts. For the Face-on view we show with an additional black dashed line the circle that was fit to the data.

the Full Width at Half Maximum (FWHM) and the second derivative of these supergaussians was used to obtain a measurement of the plasma extension in both directions. The lineouts are indicated in the second and fourth columns of Fig. 7 as the white lines. The black dashed lines indicate the regions used to integrate the lineouts, whereas the red dashed lines indicate the calculated extension of the plasma. For the Face-on images, two perpendicular lineouts were taken to obtain an average radius (shown in Fig. 7 as a black dashed circle).

The measured dimensions of the plasma during the expansion are shown in Fig. 9. We found that the radius remains constant within $10 \%$, showing that the large radius of the Be tamper compared to the Fe: $\mathrm{V}$ sample is working as expected. The target is therefore mostly expanding in the axial direction. The expansion velocity was measured to be $v=196.86 \pm 0.13 \mu \mathrm{m} \mathrm{ns}^{-1}$, which corresponds to each face of the cylinder moving at half this value $\left(v_{\text {face }}=98.43 \pm 0.07 \mu \mathrm{m} \mathrm{ns}^{-1}\right)$.
The aspect ratio $H / R$ was calculated from the data in Fig. 9. As the integration window for the XRPINH cameras is $50 \mathrm{ps}$, and for the MSPECs it is $200 \mathrm{ps}$, the average aspect ratio in each MSPEC time window was used for the spectral analysis.

\section{B. Plasma conditions}

To estimate the plasma temperature from the spectra, we used the relative intensity of the $y$-line with respect to the Li-like satellite peak on the He- $\alpha$ complex. As these lines are usually optically thin in HED conditions, this method minimizes the errors in the measurement due to optical depth effects. This can be done because the temperatures in this experiment are sufficiently low so that the Li-like satellite emission is present. This process is schematically shown in Fig. 10. In this figure, we show the spectrum from the Li-like satellites and the $y$-line for a particular time step, compared with the spectra 


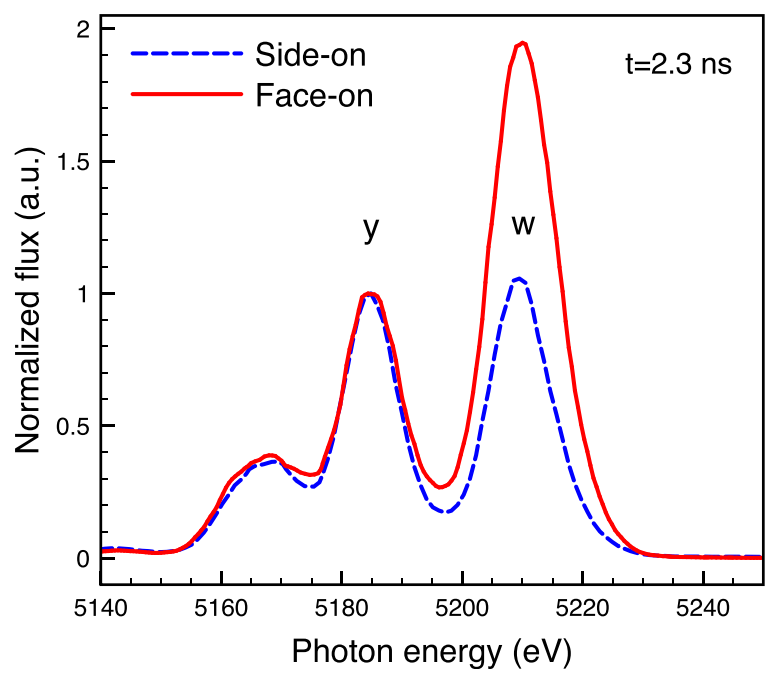

FIG. 8. Example of Face-on and Side-on spectra for V, taken from Fig. 7. These results correspond to an aspect ratio $H / R$ of $0.82(t=2.3 \mathrm{~ns})$. The spectra showed in the imaged were obtained by the same spectrometer in two subsequent shots using the two different TOs. Note the differences in the He-like lines between both views. The spectra are normalized to the peak of the $y$-line (photon energy $\sim 5185 \mathrm{eV})$.

simulated with the radiation transport code $\operatorname{CRETIN}^{54}$ for different temperatures. The spectra have been normalized to the peak of the $y$ line. In this particular example, the plasma temperature was found to be $875 \pm 25 \mathrm{eV}$.

For every shot, we calculated a temperature for the spectra from both MSPECs, finding agreement between the two views within 15\% for all shots. The measured temperature evolution is shown in Fig. 11(a).

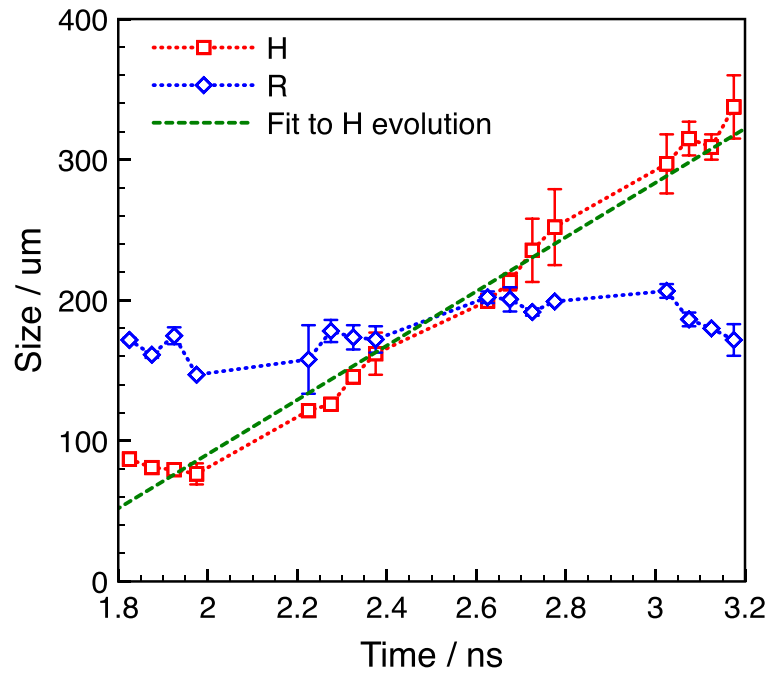

FIG. 9. Size evolution of the targets in both the axial $(H)$ and radial $(R)$ dimensions. Note how the radius stays relatively constant within $\sim 10 \%$, whereas the expansion is mostly occurring in the axial direction. The velocity of the expansion is obtained from a fit to the axial expansion (dashed green line), obtaining a value of $196.86 \pm 0.13 \mu \mathrm{m} \mathrm{ns}^{-1}$.

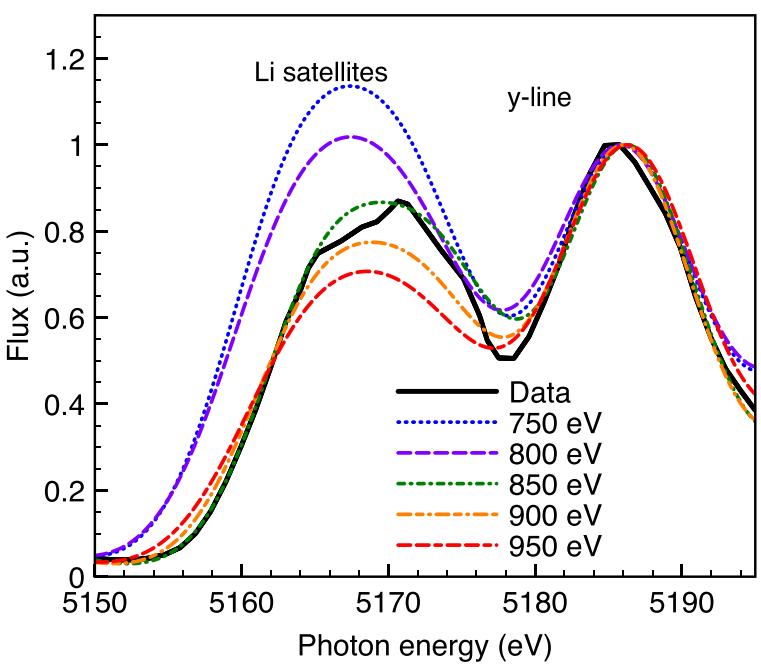

FIG. 10. Example of the method used to estimate the plasma temperature. The Lilike satellites to $y$-line ratio was compared between experiment (black solid line) and simulations for different temperatures. The density used for the simulations was obtained from the measured size from the XRPINH images. These two lines were used, as they are commonly optically thin and therefore, optical depth effects in their spectra are negligible. The temperature found in this case was $875 \pm 25 \mathrm{eV}$.

The ion density of the plasma was calculated using the size of the plasma from the XRPINH images and imposing a conservation of particle condition. These results are indicated in Fig. 11(b) as green circles. As a double check, the ion density was also obtained from the $w / y$ ratios for Face-on and Side-on views, using the method by PérezCallejo et al. . $^{53}$ This method assumes a perfectly uniform plasma cylinder and calculates the ion density from the differences in the $w / y$ ratio in the Face-on and Side-on spectra. To our knowledge, no experimental confirmation of this method has hitherto been obtained. The results are shown in Fig. 11(b) as the blue triangles. Although the spectral method does not show a clear trend for the density, which is observed in the imaging data, it retrieves the right order of magnitude for the density within error bars. However, it fails to yield a result for the last time step. This may due to the fact that so late in time the plasma could be losing its assumed cylindrical shape (as inferred from the imaging data at $3.1 \mathrm{~ns}$ in Fig. 7).

\section{C. $w / y$ line ratios}

To understand the effects of the geometry on the spectra, the plasma was simulated using a 2-dimensional radiation transfer model with the code CRETIN. ${ }^{54}$ This model was used for two things-to obtain an expected $w / y$ ratio for a perfect cylinder at the experimental conditions, and to remove the Li-like contribution in the data. An experimental $w / y$ ratio can then be obtained by integrating the flux under the remaining He- $\alpha$ lines. We found that density gradients of the order of those predicted by HYDRA had a negligible effect in the line ratios at the conditions of interest. Figure 12 shows an example of how this ratio is obtained for Face-on and Side-on emission.

In Fig. 13, we show the comparison between the $w / y$ ratios for Face-on and Side-on views, as measured in the experiment. The red 


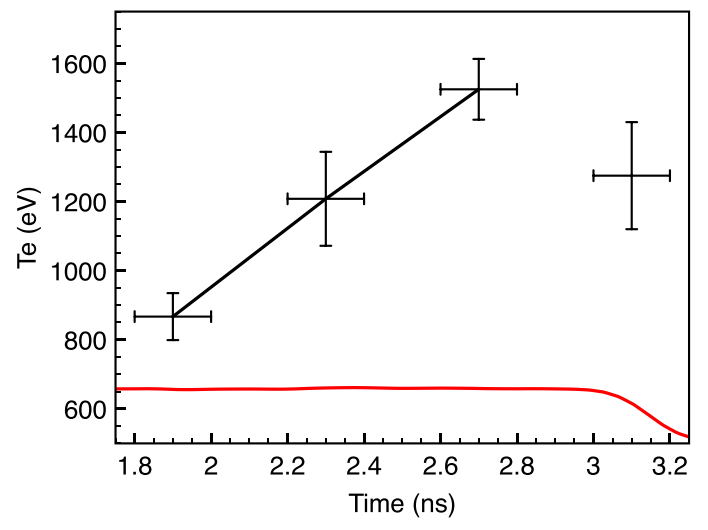

(a)

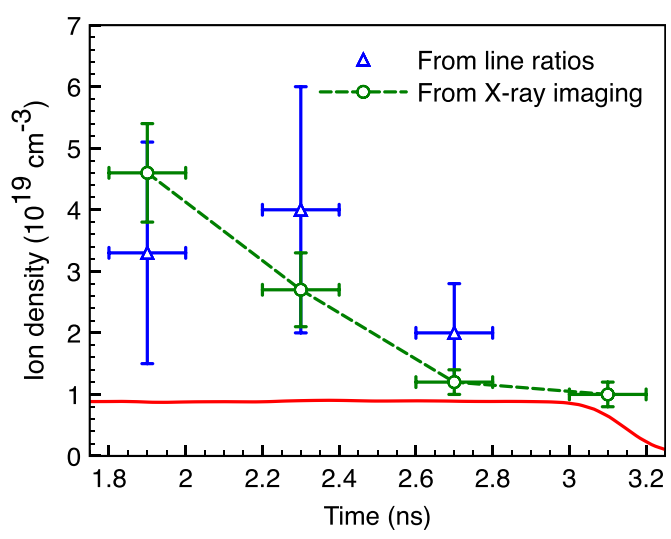

(b)

FIG. 11. (a) Temperature obtained from the $y$-line to Li satellite ratio for both spectrometers and the three shots considered. The uncertainty in the temperature corresponds to the standard deviation between shots and between Face-on and Side-on views, as well as a $50 \mathrm{eV}$ resolution in the CRETIN calculations; and the uncertainty in the time corresponds to the uncertainty in timing the framing cameras. The last point is not connected as it was obtained after the laser pulse was turned off. (b) lon density of the targets measured by two different methods. The green circles correspond to the values obtained when using the size of the targets measured by the imaging cameras, given that the total number of ions is known. The error in these data comes from the resolution of the system (due to the pinhole size, diffraction, and point-spread function of the framing cameras), as well as from differences between shots and cameras. The blue triangles correspond to the values obtained using the method described in the study by PérezCallejo et al. for plasma cylinders. ${ }^{53}$ The error values arise from the error in the line ratios (shown later in Fig. 13). The laser pulse is shown as a red line in both figures.

squares correspond to the ratio measured for Face-on view, whereas the blue circles correspond to the Side-on view results. The error bars correspond to one standard deviation and were calculated including shot-to-shot differences, as well as the differences between the two spectrometers. The error contribution from the parameters in the fit is also included. The black lines (solid and dashed) correspond to the results obtained for a perfect cylinder using our model. The solid line corresponds to the prediction for the ratio for the Face-on view, while the dashed line corresponds to the prediction for the Side-on view. The error bars in the model correspond to the uncertainty in the temperature and density of the plasma. We also show for comparison, the results predicted for a spherical plasma at the same temperature and density (gray shaded region). In this case, there are no differences between views, and this region lies between the prediction for Face-on and Side-on views of a cylinder.

Note how early in the expansion, for small aspect ratios, the obtained ratios agree with the model predictions for a perfect cylinder. For $H / R=1.15$, the geometry starts to differ from a perfect cylinder (as it can be seen in Fig. 7), and therefore, the results of simulations and experiment yield different results, with the last data point in this plot $(H / R \sim 1.7)$, as noted above, not being consistent with a cylindrical plasma.

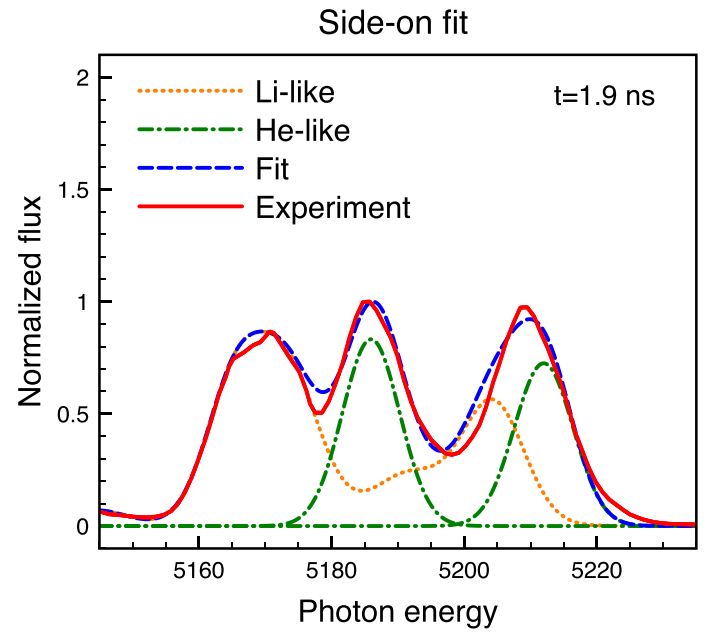

(a)
Face-on fit

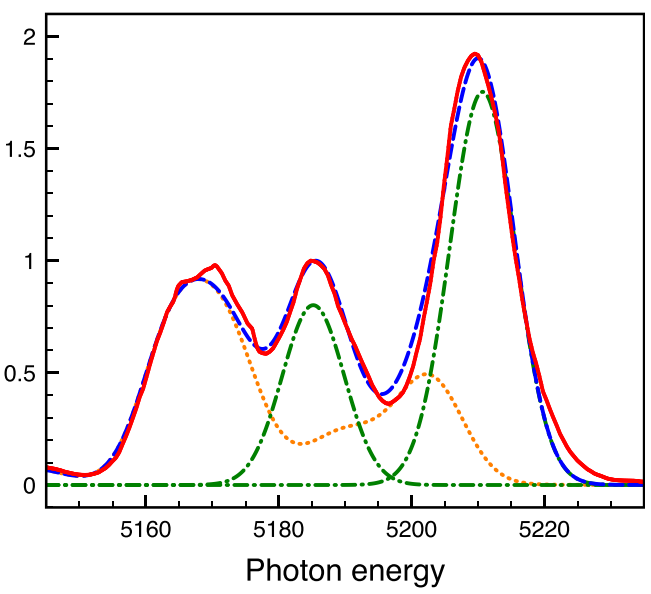

(b)

FIG. 12. Example of Face-on and Side-on fits to the spectra at $t=1.9 \mathrm{~ns}$. The Li-like emission was directly extracted from the CRETIN model for the measured temperature and density. The wly ratio was then obtained by integrating the emission under each of the He-like lines. 


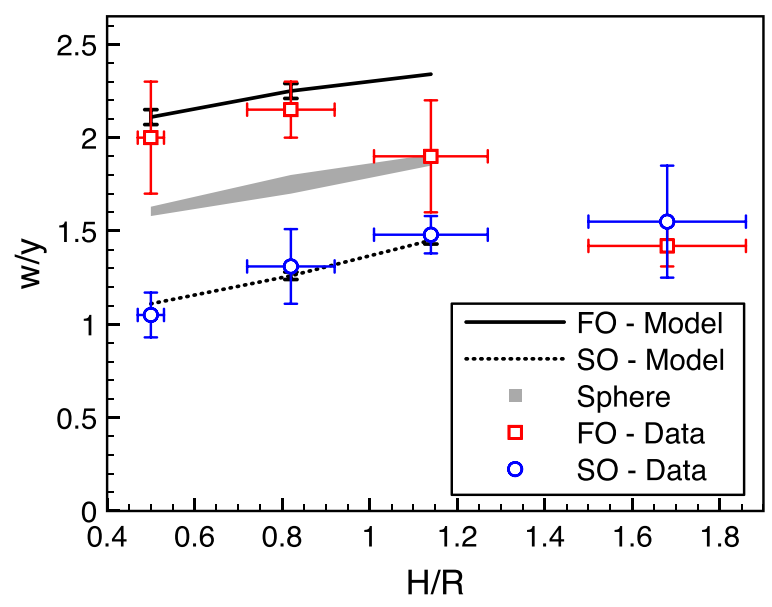

FIG. 13. Integrated w/y ratio from the experiment and simulations. Red squares and blue circles correspond to the experimental ratio for Face-on and Side-on views, respectively. The black lines correspond to the results from CRETIN simulations for Face-on (solid) and Side-on (dotted) views. The gray shaded region shows the expected result for a spherical plasma at the measured temperature and density. The cylindrical simulations are found to be in good agreement with the data for the first two data points. For $H / R=1.15$, the geometry starts to differ from a perfect cylinder (view Fig. 7), and therefore, simulations and data yield different wly ratios. The last data point in this plot $(H / R \sim 1.7)$ corresponds to a sufficiently late time that the assumption that the dot remains cylindrical is suspect, as inferred from the imaging data at $3.1 \mathrm{~ns}$ in Fig. 7.

The data show a clear difference between the spectra for Faceon and Side-on views. To understand the strength of this evidence, we performed two different statistical tests in our data. First, a $t$-test, where the null hypothesis was that there are no differences between the $w / y$ ratios for Face-on and Side-on views. This test retrieved a probability of $p=4.11 \times 10^{-5}$ of obtaining the experimental data if the null hypothesis were true. This indicates strong evidence that enhancement and suppression of the optically thick line were observed.

Second, we calculated the Bayes factor for our radiative transfer model with respect to the spherical plasma model, given our data. Bayes factors indicate the preference for one model with respect to another given a particular dataset. ${ }^{55-57}$ In our case, we obtain a value of $2 \log (K)=45.37$, where $K$ is the Bayes factor. This corresponds to a "decisive" preference for our model with respect to the spherical plasma, according to Jeffreys, ${ }^{56}$ or "very strong," using the interpretation by Kass and Raftery.

\section{CONCLUSIONS AND FUTURE WORK}

We have developed a new experimental platform for diagnosing the effects of the plasma geometry on the anisotropy of the emission of optically thick lines in non-Local Thermodynamic Equilibrium (nonLTE) plasmas at OMEGA. By using multiple target orientations and a carefully constructed laser drive configuration, we have produced selfconsistent spatial, spectral, and temporal data from cylindrical targets with geometrical aspect ratios ranging from 0.4 to 2.0 . The results are independent of the instrument response. These data enabled the characterization of the line anisotropies in the K-shell emission spectrum of mid-Z materials such as those used in dot spectroscopy experiments at NIF.
The relatively low temperatures in the experiment allowed us to use the optically thin emission from Li-like satellites as a thermometer. The obtained temperatures are consistent among shots and for different views, which is in agreement with the plasma being uniform. We have also presented the first results of ion density calculations using the He- $\alpha$ spectra of cylindrical HED plasmas. ${ }^{53}$ These results agree with the measured density from the imaging data and show the capabilities of the method in the study by Pérez-Callejo et al. as a spectroscopic diagnostics.

We have found strong evidence of enhancement and suppression of the optically thick line emission depending on the direction of emission. We have built a radiative transfer model which agrees with the observed data to a much higher degree than models which do not account for the plasma geometry. This allows for a correction in the temperatures and densities obtained from line ratios in HED or ICF experiments using dot-like plasmas as a spectral diagnostics, where the temperatures are so high that the Li-like satellites cannot be used to measure temperature.

Future work aims to spatially resolve the spectra to account for any temperature or density gradients present in the plasma. This work will also be implemented in future NIF experiments to improve the measurements of the temperature and density conditions inside a hohlraum.

\section{ACKNOWLEDGMENTS}

We thank the crew of the Omega laser at the University of Rochester Laboratory for Laser Energetics for expertly executing these experiments.

G.P.-C. and J.S.W. gratefully acknowledge support from LLNL under Grant No. B617350.

The targets were fabricated by General Atomics thanks to support from the National Nuclear Security Administration under Contract No. DE-NA0001808.

This work was performed under the auspices of the U.S. Department of Energy by the Lawrence Livermore National Laboratory under Contract No. DE-AC52-07NA27344.

\section{REFERENCES}

'E. Storm, “Approach to high compression in inertial fusion," J. Fusion Energy 7(2), 131-137 (1988).

${ }^{2}$ J. Lindl, "Development of the indirect-drive approach to inertial confinement fusion and the target physics basis for ignition and gain," Phys. Plasmas 2(11), 3933-4024 (1995).

${ }^{3}$ R. E. Olson, R. J. Leeper, A. Nobile, J. A. Oertel, G. A. Chandler, K. Cochrane, S. C. Dropinski, S. Evans, S. W. Haan, J. L. Kaae, J. P. Knauer, K. Lash, L. P. Mix, A. Nikroo, G. A. Rochau, G. Rivera, C. Russell, D. Schroen, R. J. Sebring, D. L. Tanner, R. E. Turner, and R. J. Wallace, "Shock propagation, preheat, and $\mathrm{x}$-ray burnthrough in indirect-drive inertial confinement fusion ablator materials," Phys. Plasmas 11(5), 2778-2789 (2004).

${ }^{4}$ O. A. Hurricane, D. A. Callahan, D. T. Casey, P. M. Celliers, C. Cerjan, E. L. Dewald, T. R. Dittrich, T. Döppner, D. E. Hinkel, L. F. B. Hopkins, J. L. Kline, S. Le Pape, T. Ma, A. G. MacPhee, J. L. Milovich, A. Pak, H. S. Park, P. K. Patel, B. A. Remington, J. D. Salmonson, P. T. Springer, and R. Tommasini, "Fuel gain exceeding unity in an inertially confined fusion implosion," Nature 506, 343 (2014).

${ }^{5}$ O. A. Hurricane, D. A. Callahan, D. T. Casey, E. L. Dewald, T. R. Dittrich, T. Döppner, M. A. Barrios Garcia, D. E. Hinkel, L. F. Berzak Hopkins, P. Kervin, J. L. Kline, S. L. Pape, T. Ma, A. G. MacPhee, J. L. Milovich, J. Moody, A. E. Pak, P. K. Patel, H. S. Park, B. A. Remington, H. F. Robey, J. D. Salmonson, P. 
T. Springer, R. Tommasini, L. R. Benedetti, J. A. Caggiano, P. Celliers, C. Cerjan, R. Dylla-Spears, D. Edgell, M. J. Edwards, D. Fittinghoff, G. P. Grim, N. Guler, N. Izumi, J. A. Frenje, M. Gatu Johnson, S. Haan, R. Hatarik, H. Herrmann, S. Khan, J. Knauer, B. J. Kozioziemski, A. L. Kritcher, G. Kyrala, S. A. Maclaren, F. E. Merrill, P. Michel, J. Ralph, J. S. Ross, J. R. Rygg, M. B. Schneider, B. K. Spears, K. Widmann, and C. B. Yeamans, "The high-foot implosion campaign on the National Ignition Facilitya)," Phys. Plasmas 21, 056314 (2014).

${ }^{6}$ O. A. Hurricane, D. A. Callahan, D. T. Casey, E. L. Dewald, T. R. Dittrich, T. Döppner, S. Haan, D. E. Hinkel, L. F. Berzak Hopkins, O. Jones, A. L. Kritcher, S. Le Pape, T. Ma, A. G. MacPhee, J. L. Milovich, J. Moody, A. Pak, H. S. Park, P. K. Patel, J. E. Ralph, H. F. Robey, J. S. Ross, J. D. Salmonson, B. K. Spears, P. T. Springer, R. Tommasini, F. Albert, L. R. Benedetti, R. Bionta, E. Bond, D. K. Bradley, J. Caggiano, P. M. Celliers, C. Cerjan, J. A. Church, R. Dylla-Spears, D. Edgell, M. J. Edwards, D. Fittinghoff, M. A. Barrios Garcia, A. Hamza, R. Hatarik, H. Herrmann, M. Hohenberger, D. Hoover, J. L. Kline, G. Kyrala, B. Kozioziemski, G. Grim, J. E. Field, J. Frenje, N. Izumi, M. Gatu Johnson, S. F. Khan, J. Knauer, T. Kohut, O. Landen, F. Merrill, P. Michel, A. Moore, S. R. Nagel, A. Nikroo, T. Parham, R. R. Rygg, D. Sayre, M. Schneider, D. Shaughnessy, D. Strozzi, R. P. J. Town, D. Turnbull, P. Volegov, A. Wan, K. Widmann, C. Wilde, and C. Yeamans, "Inertially confined fusion plasmas dominated by alpha-particle self-heating,” Nat. Phys. 12, 800-806 (2016).

${ }^{7}$ R. Betti, A. R. Christopherson, B. K. Spears, R. Nora, A. Bose, J. Howard, K. M. Woo, M. J. Edwards, and J. Sanz, "Alpha heating and burning plasmas in inertial confinement fusion,” Phys. Rev. Lett. 114, 255003 (2015).

${ }^{8}$ L. C. Jarrott, B. Bachmann, T. Ma, L. R. Benedetti, F. E. Field, E. P. Hartouni, R. Hatarik, N. Izumi, S. F. Khan, O. L. Landen et al., "Thermal temperature measurements of inertial fusion implosions," Phys. Rev. Lett. 121(8), 085001 (2018).

${ }^{9} \mathrm{~L}$. Heroux, "A spectroscopic determination of electron temperature in a high temperature plasma," Proc. Phys. Soc. 83(1), 121 (1964).

${ }^{10}$ K. P. Dere, H. E. Mason, K. G. Widing, and A. K. Bhatia, "XUV electron density diagnostics for solar flares," Astrophys. J. Suppl. Ser. 40, 341-364 (1979).

${ }^{11} \mathrm{E}$. Landi and M. Landini, "Simultaneous temperature and density diagnostics of optically thin plasmas," Astron. Astrophys. 327, 1230-1241 (1997).

${ }^{12}$ J. M. Laming, U. Feldman, U. Schühle, P. Lemaire, W. Curdt, and K. Wilhelm, "Electron density diagnostics for the solar upper atmosphere from spectra obtained by sumer/soho," Astrophys. J. 485(2), 911 (1997).

${ }^{13}$ V. Polito, G. Del Zanna, J. Dudík, H. E. Mason, A. Giunta, and K. K. Reeves, "Density diagnostics derived from the oand s intercombination lines observed by iris," Astron. Astrophys. 594, A64 (2016).

${ }^{14}$ R. L. Porter and G. J. Ferland, "Revisiting he-like x-ray emission-line plasma diagnostics," Astrophys. J. 664(1), 586 (2007).

${ }^{15} \mathrm{~A}$. H. Gabriel, "Dielectronic satellite spectra for highly-charged helium-like ion lines," Mon. Not. R. Astron. Soc. 160, 99-119 (1972).

${ }^{16} \mathrm{~A}$. H. Gabriel and C. Jordan, "Interpretation of solar helium-like ion line intensities," Mon. Not. R. Astron. Soc. 145(2), 241-248 (1969).

${ }^{17}$ G. R. Blumenthal, G. W. F. Drake, and W. H. Tucker, "Ratio of line intensities in helium-like ions as a density indicator,” Astrophys. J. 172, 205 (1972).

${ }^{18}$ F. P. Keenan, W. A. Feibelman, and K. A. Berrington, "Improved calculations for the C III 1907,1909 and Si III 1883,1892 electron density sensitive emissionline ratios, and a comparison with IUE observations," Astrophys. J. 389, 443-446 Apr. (1992).

${ }^{19}$ D. Porquet, R. Mewe, J. Dubau, A. J. J. Raassen, and J. S. Kaastra, "Line ratios for helium-like ions: Applications to collision-dominated plasmas*," Astron. Astrophys. 376(3), 1113-1122 (2001).

${ }^{20}$ J.-U. Ness, R. Mewe, J. H. M. M. Schmitt, A. J. J. Raassen, D. Porquet, J. S. Kaastra, R. L. J. van der Meer, V. Burwitz, and P. Predehl, "Helium-like triplet density diagnostics-applications to chandra-letgs $\mathrm{x}$-ray observations of capella and procyon," Astron. Astrophys. 367(1), 282-296 (2001).

${ }^{21}$ R. Mewe, A. J. J. Raassen, J. J. Drake, J. S. Kaastra, R. L. J. van der Meer, and D. Porquet, "Chandra-letgs x-ray observations of capella," Astron. Astrophys. 368, 888-900 (2001).

${ }^{22}$ J.-U. Ness, J. H. M. M. Schmitt, V. Burwitz, R. Mewe, A. J. J. Raassen, R. L. J. van der Meer, P. Predehl, and A. C. Brinkman, "Coronal density diagnostics with helium-like triplets: Chandra-letgs observations of algol, capella, procyon, i, n a\&b, ux ari, ad leo, yy gem, and hr9," Astron. Astrophys. 394(3), 911-926 (2002).

${ }^{23}$ D. Porquet, J. Dubau, and N. Grosso, "He-like ions as practical astrophysical plasma diagnostics: From stellar coronae to active galactic nuclei," Space Sci. Rev. 157, 103-134 (2010).

${ }^{24} \mathrm{~A}$. K. Pradhan, "On the systematics of line ratios along the helium isoelectronic sequence," Astrophys. J. 263, 477-482 (1982).

${ }^{25}$ R. S. Marjoribanks, M. C. Richardson, P. A. Jaanimagi, and R. Epstein, "Electron-temperature measurement in laser-produced plasmas by the ratio of isoelectronic line intensities," Phys. Rev. 46, R1747 (1992).

${ }^{26}$ C. A. Back, D. H. Kalantar, R. L. Kauffman, R. W. Lee, B. K. MacGowan, D. S. Montgomery, L. V. Powers, T. D. Shepard, G. F. Stone, and L. J. Suter, "Measurements of electron temperature by spectroscopy in hohlraum targets," Phys. Rev. Lett. 77(21), 4350 (1996).

${ }^{27}$ M. A. Barrios, D. A. Liedahl, M. B. Schneider, O. Jones, G. V. Brown, S. P. Regan, K. B. Fournier, A. S. Moore, J. S. Ross, O. Landen, R. L. Kauffman, A. Nikroo, J. Kroll, J. Jaquez, H. Huang, S. B. Hansen, D. A. Callahan, D. E. Hinkel, D. Bradley, and J. D. Moody, "Electron temperature measurements inside the ablating plasma of gas-filled hohlraums at the National Ignition Facility," Phys. Plasmas 23(5), 056307 (2016).

${ }^{28}$ J. D. Kilkenny, R. W. Lee, M. H. Key, and J. G. Lunney, "X-ray spectroscopic diagnosis of laser-produced plasmas, with emphasis on line broadening," Phys. Rev. A. 22, 2746-2760 (1980).

${ }^{29}$ J. P. Apruzese, K. G. Whitney, J. Davis, and P. C. Kepple, "K-shell line ratios and powers for diagnosing cylindrical plasmas of neon, aluminum, argon, and titanium," J. Quant. Spectrosc. Radiat. Transfer 57(1), 41-61 (1997).

30 J. P. Apruzese, D. Duston, and J. Davis, "K-shell aluminum resonance line ratios for plasma diagnosis using spot spectroscopy," J. Quant. Spectrosc. Radiat. Transfer 36(4), 339-344 (1986).

${ }^{31}$ J. D. Kilkenny, "Recent diagnostic developments at nova (invited)," Rev. Sci. Instrum. 63(10), 4688-4694 (1992).

${ }^{32}$ T. D. Shepard, C. A. Back, D. H. Kalantar, R. L. Kauffman, C. J. Keane, D. E. Klem, B. F. Lasinski, B. J. MacGowan, L. V. Powers, L. J. Suter, R. E. Turner, B. H. Failor, and W. W. Hsing, "Isoelectronic x-ray spectroscopy to determine electron temperatures in lon-scale-length inertial-confinement-fusion plasmas," Phys. Rev. E 53(5), 5291 (1996).

${ }^{33}$ G. H. Miller, E. I. Moses, and C. R. Wuest, "The National Ignition Facility," Opt. Eng. 43, 43-43-13 (2004).

${ }^{34}$ M. A. Barrios, J. D. Moody, L. J. Suter, M. Sherlock, H. Chen, W. Farmer, J. Jaquez, O. Jones, R. L. Kauffman, J. D. Kilkenny, J. Kroll, O. L. Landen, D. A. Liedahl, S. A. Maclaren, N. B. Meezan, A. Nikroo, M. B. Schneider, D. B. Thron, K. Widmann, and G. Pérez-Callejo, "Developing an experimental basis for understanding transport in NIF hohlraum plasmas," Phys. Rev. Lett. 121, 095002 (2018).

${ }^{35}$ A. K. Bhatia and S. O. Kastner, "The optically thick Fe xvii spectrum: X-ray, extreme-ultraviolet, and forbidden line ratios," Astrophys. J. 516(1), 482 (1999).

${ }^{36}$ A. K. Bhatia and J. L. R. Saba, "Resonance scattering of Fe xvii x-ray and extreme-ultraviolet lines," Astrophys. J. 563(1), 434 (2001).

${ }^{37}$ S. O. Kastner and A. K. Bhatia, "Optically thin and thick Fe xv spectrum: Effect of self-absorption on the $284.16 \AA$ resonance line," Astrophys. J. 553(1), 421 (2001).

${ }^{38}$ F. M. Kerr, S. J. Rose, J. S. Wark, and F. P. Keenan, "Enhancement of optically thick to thin line intensities in solar and stellar coronal plasmas through radiative transfer effects: An angularly resolved study," Astrophys. J. 613, L181-L184 (2004)

${ }^{39}$ F. M. Kerr, S. J. Rose, and J. S. Wark, "An analytic geometry-variant approach to line ratio enhancement above the optically thin limit," Astrophys. J. 629, 1091 (2005).

${ }^{40}$ F. M. Kerr, A. Gouveia, O. Renner, S. J. Rose, H. A. Scott, and J. S. Wark, "Line radiation effects in laboratory and astrophysical plasmas," J. Quant. Spectrosc. Radiat. Transfer 99, 363-369 (2006).

${ }^{41}$ P. A. M. Dirac, "Approximate rate of neutron multiplication for a solid of arbitrary shape and uniform density, I: General theory," in The Collected Works of P. A. M. Dirac 1924-1948 (Oxford University Press, 1995), pp. 1115-1128.

${ }^{42}$ P. A. M. Dirac, K. Fuchs, R. Peierls, and P. Preston, "Approximate rate of neutron multiplication for a solid of arbitrary shape and uniform density, II: 
Application to the oblate spheroid, hemisphere and oblate hemispheroid," in The Collected Works of P. A. M. Dirac 1924-1948 (Oxford University Press, 1995), pp. 1129-1145.

${ }^{43} \mathrm{P}$. Hatfield, "Using line intensity ratios to determine the geometry of plasma in stars via their apparent areas," High Energy Density Phys. 6(3), 301-304 (2010).

${ }^{44}$ D. M. Chambers, P. A. Pinto, J. Hawreliak, I. R. Al'Miev, A. Gouveia, P. Sondhauss, E. Wolfrum, J. S. Wark, S. H. Glenzer, R. W. Lee, P. E. Young, O. Renner, R. S. Marjoribanks, and S. Topping, "K-shell spectroscopy of an independently diagnosed uniaxially expanding laser-produced aluminum plasma," Phys. Rev. E 66, 026410 (2002).

${ }^{45}$ T. R. Boehly, D. L. Brown, R. S. Craxton, R. L. Keck, J. P. Knauer, J. H. Kelly, T. J. Kessler, S. A. Kumpan, S. J. Loucks, S. A. Letzring, F. J. Marshall, R. L. McCrory, S. F. B. Morse, W. Seka, J. M. Soures, and C. P. Verdon, "Initial performance results of the omega laser system," Opt. Commun. 133(1), 495-506 (1997).

${ }^{46}$ W. J. Gray, M. E. Foord, M. B. Schneider, M. A. Barrios, G. V. Brown, R. F. Heeter, L. C. Jarrott, D. A. Liedahl, E. V. Marley, C. W. Mauche, and K. Widmann, "Investigation of the hydrodynamics and emission of a laser heated tamped high-z target," Phys. Plasmas 25, 062702 (2018).

${ }^{47}$ M. M. Marinak, R. E. Tipton, O. L. Landen, T. J. Murphy, P. Amendt, S. W. Haan, S. P. Hatchett, C. J. Keane, R. McEachern, and R. Wallace, "Threedimensional simulations of nova high growth factor capsule implosion experiments," Phys. Plasmas 3(5), 2070-2076 (1996).

${ }^{48}$ G. E. Kemp, L. C. Jarrott, E. V. Marley, R. F. Heeter, D. A. Liedahl, C. W. Mauche, P. K. Patel, M. B. Schneider, K. Widmann, and M. E. Foord, "Generating uniform, non-equilibrium, mid- to high-Z plasmas for radiative properties studies at the Omega laser facility," in APS Meeting Abstracts, October 2017, p. YP11.014.

${ }^{49}$ R. F. Heeter, J. A. Emig, K. B. Fournier, S. B. Hansen, M. J. May, and B. K. F. Young, "X-ray spectroscopy with elliptical crystals and face-on framing cameras," Rev. Sci. Instrum. 75, 3762-3764 (2004).

${ }^{50}$ K. M. Campbell, F. A. Weber, E. L. Dewald, S. H. Glenzer, O. L. Landen, R. E. Turner, and P. A. Waide, "Omega dante soft $\mathrm{x}$-ray power diagnostic component calibration at the national synchrotron light source," Rev. Sci. Instrum. 75(10), 3768-3771 (2004)

${ }^{51}$ C. Sorce, J. Schein, F. Weber, K. Widmann, K. Campbell, E. Dewald, R. Turner, O. Landen, K. Jacoby, P. Torres, and D. Pellinen, "Soft x-ray power diagnostic improvements at the omega laser facility," Rev. Sci. Instrum. 77(10), 10E518 (2006).

${ }^{52}$ M. J. May, K. Widmann, C. Sorce, H.-S. Park, and M. B. Schneider, "Uncertainty analysis technique for omega dante measurements," Rev. Sci. Instrum. 81(10), 10E505 (2010).

${ }^{53}$ G. Pérez-Callejo, D. A. Liedahl, M. B. Schneider, S. J. Rose, and J. S. Wark, "The use of geometric effects in diagnosing ion density in ICF-related dot spectroscopy experiments," High Energy Density Phys. 30, 45-51 (2019).

${ }^{54}$ H. A. Scott, "CRETIN-A radiative transfer capability for laboratory plasmas," J. Quant. Spectrosc. Radiat. Transfer 71(2), 689-701 (2001).

${ }^{55} \mathrm{H}$. Jeffreys, "Some tests of significance, treated by the theory of probability," Math. Proc. Cambridge Philos. Soc. 31(2), 203-222 (1935).

${ }^{56} \mathrm{H}$. Jeffreys, The Theory of Probability (Oxford, 1961).

${ }^{57}$ R. E. Kass and A. E. Raftery, "Bayes factors," J. Am. Stat. Assoc. 90, 773-795 (1995). 